\section{RELIEVE}

ISSN: 1134-4032
e-Journal of Educational Research,

Assessment and Evaluation

\title{
Validación del cuestionario de atribuciones para la detección de coincidencias en trabajos académicos
}

\author{
Validation of a questionnaire of attributions to the detection of coincidences in academic work
}

\author{
Muñoz-Cantero, Jesús-Miguel ${ }^{(1)}$; Rebollo-Quintela, Nuria ${ }^{(1)}$; Mosteiro-García, Josefa ${ }^{(2)}$ \& \\ Ocampo-Gómez, Camilo-Isaac ${ }^{(3)}$
}

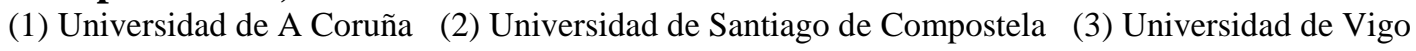

\begin{abstract}
Plagiarism as a topic of research in the university context has not ceased to be a constant concern due to its multiple repercussions. This paper describes the process followed in the validation of the Questionnaire of attributions for the detection of coincidences in academic works (CUDECO), whose purpose is to evaluate the relevance that to cite correctly and to detect the causes that lead to commit plagiarism. From a sample of 2,331 students, an analysis of reliability as internal consistency and an exploratory factorial analysis (AFE) of the instrument is carried out. The analysis of reliability leads to propose a readjustment in the questionnaire initially designed. The factorial study suggests a structure configured by five factors: concept of plagiarism and its types (partial and total), causes that motivate the commission of it both internal (specific to the subject) and external (outside the subject) and the attitudes of the peer group towards plagiarism, findings in the line of research previous.
\end{abstract}

Reception Date 2018 December 18

Approval Date 2019 March 15

Publication Date:

2019 March 18

Keywords: Validation, factorial analysis, plagiarism, university students, dishonest practices

\begin{abstract}
Resumen
El plagio como tema de investigación en el contexto universitario no ha cesado de ser una preocupación constante debido a sus múltiples repercusiones. El presente estudio describe el proceso seguido en la validación del Cuestionario de atribuciones para la detección de coincidencias en trabajos académicos (CUDECO), cuya finalidad es evaluar la relevancia que tiene citar correctamente y detectar las causas que llevan a cometer plagio. A partir de una muestra de 2,331 estudiantes, se realiza un análisis de la fiabilidad como consistencia interna y un análisis factorial exploratorio (AFE) del instrumento. El análisis de la fiabilidad lleva a proponer un reajuste en el cuestionario inicialmente diseñado y el estudio factorial sugiere una estructura configurada por cinco factores: concepto de plagio y sus tipos (parcial y total), causas que motivan la comisión del mismo tanto internas (propias del sujeto) como externas (ajenas al sujeto) y las actitudes del grupo de iguales hacia el plagio, hallazgos en la línea de investigaciones previas.
\end{abstract}

Palabras clave: Validación, análisis factorial, plagio, estudiantes universitarios, prácticas deshonestas

La presencia en el ámbito académico y científico de prácticas deshonestas está siendo objeto de controversia en el plano social, por sus repercusiones, y también un foco de estudio a nivel jurídico, ético y educativo (Ferreira \& Persike, 2018). En este último ámbito, Mavrinac, Brumini, Bilić-Zulle y Petrovečki, (2010) aluden a que estas prácticas incluyen cualquier tipo de engaño que comprometa el proceso de enseñanza- aprendizaje y la integridad académica de la institución, siendo el plagio el más perpetrado. Respecto a éste, no existe unanimidad a la hora de definirlo, debido a su universalidad, multidimensionalidad, multicausalidad y a los determinantes culturales del mismo. Esta falta de consenso, en la educación universitaria, viene motivada también por su magnitud ya que abarca desde pequeñas oraciones sin citación hasta la copia de una obra completa 
(Bennett, 2005), o como señala Hawley (1984), el plagio cubre un continuo entre "paráfrasis descuidadas hasta la copia intencional del trabajo de otra persona literalmente sin crédito a la fuente” (p.35). En general, podemos caracterizarlo como la copia o paráfrasis de un fragmento del trabajo de alguien sin citar el original (Park, 2003), incluyendo también el autoplagio, es decir, entregar nuevamente trabajos académicos ya evaluados (Bretag \& Mahmud, 2009).

La magnitud de este fenómeno se refleja en su universalidad con estudios en: Reino Unido (Bennett, 2005; Cheung, Stupple, \& Elander, 2017), Rumanía (Popoveniuc, 2018), México (Guerrero Sánchez, Mercado Yebra, \& Ibarra, 2017), Estados Unidos (Martin, Rao, \& Sloan, 2011), Australia (Devlin \& Gray, 2007; Howard, Ehrich, \& Walton, 2014), Irán (Amiri \& Razmjoo, 2016; Poorolajal, Cheraghi, Irani, Cheragui, \& Mirfakhraei, 2012), Turquía (Akbulut et al., 2008), China (Hu \& Sun,
2016), España (Gómez-Espinosa, Francisco, \& Moreno-Ger, 2016; Sureda, Comas, \& Morey, 2009; Sureda-Negre, Comas-Forgas, \& OliverTrobat, 2015),... donde se ofrecen cifras alarmantes de haber cometido plagio por lo menos una vez en la vida, que van desde el 83\% referenciado por Bilic-Zulle, Frkovic, Turk, Azman y Petrovecki (2005) al 38\% que se constata en el trabajo de Poorolajal et al. (2012). Así mismo, nos encontramos con investigaciones que muestran diferencias interculturales: comparaciones entre estudiantado alemán y esloveno (Jereb et al., 2018), o australiano y chino (Ehrich, Howard, $\mathrm{Mu}$, \& Bokosmaty, 2016).

La multidimensionalidad y la multicausalidad del tema se refleja en la atención que se presta a los factores tanto de tipo contextual como individual en varias investigaciones en las que se han diseñado, aplicado y estandarizando instrumentos de medida centrados en el plagio (tabla 1).

Tabla 1 - Instrumentos de medida de plagio e integridad académica

\begin{tabular}{|c|c|c|c|}
\hline Instrumentos & Autoría & $\mathrm{N}^{0}$ Items & Dimensiones/subescalas \\
\hline Plagiarism Attitude Scale & Harris (2001) & 12 ítems & Instrumento unidimensional \\
\hline $\begin{array}{l}\text { Student Authorship } \\
\text { Questionnaire (SAQ) }\end{array}$ & $\begin{array}{l}\text { Pittam, Elander, } \\
\text { Lusher, Fox, \& Payne } \\
\text { (2009) }\end{array}$ & 18 ítems & $\begin{array}{l}\text { Confianza en la escritura } \\
\text { Comprensión en la escritura } \\
\text { Conocimientos para evitar el plagio }\end{array}$ \\
\hline $\begin{array}{c}\text { Attitudes Toward Plagiarism } \\
(A T P)\end{array}$ & $\begin{array}{l}\text { Mavrinac, Brumini, } \\
\text { Bilić-Zulle, \& } \\
\text { Petrovečki (2010) }\end{array}$ & 29 ítems & $\begin{array}{l}\text { Actitudes positivas ante el plagio } \\
\text { Actitudes negativas } \\
\text { Normas subjetivas ante el plagio }\end{array}$ \\
\hline $\begin{array}{c}\text { Survey on academic integrity } \\
\text { among students at the } \\
\text { University of the Balearic } \\
\text { Islands }\end{array}$ & $\begin{array}{l}\text { Comas-Forgas \& } \\
\text { Sureda-Negre (2010) }\end{array}$ & 41 ítems & $\begin{array}{l}\text { Causas atribuibles a características o } \\
\text { comportamientos del personal docente y } \\
\text { aspectos metodológicos del proceso de } \\
\text { enseñanza-aprendizaje } \\
\text { Causas atribuibles a características, } \\
\text { comportamientos y creencias de los } \\
\text { estudiantes } \\
\text { Causas atribuibles a la facilidad de acceso } \\
\text { ofrecido por la Internet y TIC }\end{array}$ \\
\hline $\begin{array}{c}\text { Knowledge, Attitude And } \\
\text { Practice Questionnaire } \\
\text { (KAP) }\end{array}$ & $\begin{array}{l}\text { Poorolajal, Cheraghi, } \\
\text { Irani, Cheragui, \& } \\
\text { Mirfakhraei (2012) }\end{array}$ & 26 ítems & $\begin{array}{l}\text { Preguntas relativas a conocimientos sobre el } \\
\text { plagio } \\
\text { Actitudes hacia el plagio } \\
\text { Actitudes hacia la comisión de plagio }\end{array}$ \\
\hline $\begin{array}{l}\text { Adaptación del Attitudes } \\
\text { Toward Plagiarism (ATP) }\end{array}$ & $\begin{array}{l}\text { Howard, Ehrich, \& } \\
\text { Walton (2014) }\end{array}$ & 36 ítems & $\begin{array}{l}\text { Factores que agravan el plagio } \\
\text { Justificaciones ante el plagio } \\
\text { Severidad y sanciones }\end{array}$ \\
\hline \multirow[t]{2}{*}{$\begin{array}{l}\text { Student Attitudes and } \\
\text { Beliefs about Authorship } \\
\text { Scale (SABAS) }\end{array}$} & $\begin{array}{l}\text { Cheung, Stupple, \& } \\
\text { Elander (2017) }\end{array}$ & 17 ítems & Instrumento unidimensional \\
\hline & $\begin{array}{l}\text { Cebrián-Robles, Raposo- } \\
\text { Rivas, Cebrián-de-la- } \\
\text { Serna, \& Sarmiento- } \\
\text { Campos (2018) }\end{array}$ & 85 ítems & $\begin{array}{l}\text { Plagio y sus motivaciones } \\
\text { Soluciones para evitar el plagio } \\
\text { Medidas a tomar en caso de plagio }\end{array}$ \\
\hline
\end{tabular}


Una de las aproximaciones se centra en su estudio mediante el Student Authorship Questionnaire (SAQ) de Pittam, Elander, Lusher, Fox, \& Payne (2009), cuestionario validado de 18 ítems con tres escalas de medida de las actitudes hacia la autoría (confianza en la escritura, comprensión en la escritura y conocimientos para evitar el plagio); posteriormente, Cheung et al. (2017) construyeron y validaron una escala tomando como referencia el SAQ denominada Student Attitudes and Beliefs about Authorship Scale (SABAS) compuesta por 17 ítems agrupados en tres factores.

Bennett (2005) confirma dos modelos de medida sobre los determinantes de cometer mayor o menor plagio, siendo estos: la posición ética, la efectividad de las habilidades de estudio personal, las actitudes hacia el plagio, el rendimiento académico, la presión de los padres, la situación financiera, la orientación hacia los objetivos, la integración académica, el miedo al fracaso, el miedo a las sanciones en el caso de ser detectados y una excelente relación con el profesorado. Profundizando más, encontramos el estudio de Mavrinac et al. (2010) en el que se valida un cuestionario de actitudes hacia el plagio (Attitudes Toward Plagiarism, ATP) compuesto por 29 ítems divididos en tres factores: actitudes positivas (aprobación y justificación del comportamiento), actitudes negativas (condena y desaprobación) y normas subjetivas ante el plagio (pensamiento común sobre su prevalencia y aceptación); por su parte Howard et al. (2014) realizan una validación de la versión modificada de la escala de Mavrinac et al. (2010) mediante el modelo de Rasch. Otro instrumento estandarizado es el Knowledge, Attitude And Practice Questionnaire (KAP) compuesto por 26 preguntas relativas a conocimientos sobre el plagio (9), actitudes hacia él (9) y la comisión de plagio (8). Por último, nos encontramos con la escala unidimensional de Ehrich et al. (2016) que, a través de 12 cuestiones, analiza las actitudes hacia el plagio desde las más permisivas a las más restrictivas.
A nivel nacional, destaca el estudio de Comas-Forgas \& Sureda-Negre (2010), en el que se especifica la validación del Survey on academic integrity among students at the University of the Balearic Islands, compuesto por 41 ítems que detallan las causas del plagio principalmente en tres aspectos: "causas atribuibles a características o comportamientos del personal docente $y$ aspectos metodológicos del proceso de enseñanza-aprendizaje; causas atribuibles a características, comportamientos y creencias de los estudiantes, causas atribuibles a la facilidad de acceso ofrecido por la Internet y TIC” (p. 220). En publicaciones posteriores, validan instrumentos para la detección del plagio en estudiantes de Enseñanza Secundaria y Bachillerato (Sureda-Negre et al., 2015). Por último, Cebrián-Robles et al. (2018), partiendo de instrumentos previamente validados (Comas-Forgas \& Sureda-Negre, 2010; Finn \& Frone, 2004) diseñan un cuestionario a fin de determinar el modelo subyacente entre las causas de plagio (concepto, parte, todo, locus interno, locus externo y desinterés).

En suma, se puede afirmar que los instrumentos diseñados pretenden determinar, en gran medida, la génesis de estas irregularidades éticas, teniendo en cuenta diferentes aspectos que influyen en la comisión de plagio entre los que destacan: "factores personales del alumnado, factores institucionales, factores ligados a la docencia y factores externos a la práctica educativa” (Sureda et al., 2015, p. 105).

\section{Método}

\section{Objetivo}

El objetivo de este trabajo es validar el Cuestionario de atribuciones para la detección de coincidencias en trabajos académicos (CUDECO) diseñado para evaluar la relevancia que tiene citar correctamente y detectar las causas que llevan a cometer plagio.

\section{Muestra}

La muestra, no probabilística e intencional, está conformada por un total de 2,331 estudiantes, $83.3 \%$ mujeres y $16.7 \%$ hombres, 
con una edad media de 21 años $(\mathrm{M}=21.68$, DT = 3.79), que cursan durante el año académico 2017/2018 estudios de Grado (92.4\%) Máster (7.3\%) y Doctorado (0.3\%) en las tres universidades públicas del Sistema Universitario Gallego (A Coruña, Santiago de Compostela y Vigo).

\section{Instrumento}

A partir de la revisión de la literatura y del análisis exhaustivo de instrumentos validados (Cebrián-Robles et al., 2018; Comas-Forgas \& Sureda-Negre, 2010; Ehrich et al.,2016; Mavrinac et al.,2010) se construyó el Cuestionario de atribuciones para la detección de coincidencias en trabajos académicos (CUDECO) configurado por un total de 59 preguntas, 9 referidas a las variables de identificación del sujeto y 50 ítems de escala tipo Likert con 7 alternativas de respuestas (siendo 1 totalmente en desacuerdo y 7 totalmente de acuerdo) agrupados en cinco dimensiones.

La primera dimensión ¿Qué utilidad tiene para ti citar?, está formada por 8 ítems, que valoran la utilidad que tiene para el alumnado citar. La segunda A lo largo de la carrera... (8 ítems), y tercera Causas que te han motivado a realizar las acciones anteriores (13 ítems) agrupan un total de 21 ítems, con los que se busca obtener información acerca de si el alumnado ha cometido plagio a lo largo de la carrera y las causas que le han motivado a ello. La cuarta dimensión, El profesorado..., comprende un total de 11 ítems relacionados con las acciones llevadas a cabo por el profesorado para que el alumnado no cometa plagio. Finalmente, los 10 ítems que configuran la dimensión Creo que mis compañeros/as están relacionados con la opinión del estudiantado acerca de la percepción que tienen sobre la realización de prácticas deshonestas por parte de sus iguales.

El cuestionario fue sometido a validación por seis jueces expertos del área de Métodos de Investigación y Diagnóstico en Educación y aplicado a una muestra piloto de 128 estudiantes de la Facultad de Ciencias de la Educación de la Universidad de A Coruña (Rebollo-Quintela, Espiñeira-Bellón, \& Muñoz-Cantero, 2017).
Procedimiento de recogida y análisis de los datos

La administración de los cuestionarios a las personas participantes fue mediante dos vías: a) en formato papel a través de encuestadores y encuestadoras que acudieron a principios del curso 2017/2018 a aulas de los diferentes títulos de Grado y Máster, y b) en formato electrónico mediante la versión online del instrumento para el alumnado de doctorado.

El tratamiento de la información se realizó con el paquete estadístico IBM SPSS Statistics en su versión 24.0. Para llevar a cabo la validación del instrumento se calculó la fiabilidad a través del análisis de consistencia interna mediante el coeficiente Alfa de Cronbach y el análisis de la capacidad de discriminación de los ítems a través del índice de homogeneidad. El estudio de la validez se realizó mediante un análisis factorial exploratorio (AFE) con extracción de componentes principales y posterior rotación Varimax.

\section{Resultados}

\section{Análisis de la fiabilidad}

El Coeficiente Alpha de Cronbach, basado en la correlación interelementos promedio, asume que los ítems miden un mismo constructo y están altamente correlacionados (Cronbach, 1951). En este caso, el valor obtenido de fiabilidad es elevado pues el coeficiente Alpha de Cronbach es igual a .879 .

Posteriormente, se procedió al análisis de la capacidad de discriminación de los ítems, a través del cálculo del índice de homogeneidad, definido como el coeficiente de correlación de Pearson entre la puntuación en el ítem y la suma de las puntuaciones en los restantes ítems. Resultado de ello, fueron eliminados 17 que mostraban valores inferiores a .2 (tabla 2), es decir, el ítem Lo considero un desafío al profesorado, a la autoridad de la dimensión Causas que te han motivado a realizar las acciones anteriores y todos los correspondientes a la dimensión ¿Qué utilidad tiene para ti citar? y El Profesorado... 
Muñoz-Cantero, Jesús-Miguel; Rebollo-Quintela, Nuria; Mosteiro-García, Josefa, \& Ocampo-Gómez, Camilo-Isaac (2019). Validación del cuestionario de atribuciones para la detección de coincidencias en trabajos académicos. RELIEVE, 25(1), art. 4. doi: http://doi.org/10.7203/relieve.25.1.13599

Tabla 2 - Valores de los índices de homogeneidad de cada ítem y del índice de fiabilidad del cuestionario

\section{¿QUE UTILIDAD TIENE PARA TI CITAR?}

Correlación total a de Cronbach de elementos si se suprime el corregida item

Sirve como requisito necesario en la realización de trabajos académicos

Sirve para demostrar que he aprendido de forma adecuada

Sirve para dar un matiz científico a mis trabajos

Sirve para reconocer el mérito de los/las autores/as originales

Sirve para remitir a las fuentes originales

Sirve para apoyar mis argumentos

Sirve para generar discusiones o debates a partir de lo citado

Sirve para demostrar que puedo generar nueva información o ideas a partir de lo citado

\section{A LO LARGO DE LA CARRERA}

He entregado algún trabajo realizado por otros/as en cursos anteriores

He copiado partes de trabajos entregados en cursos anteriores para un trabajo nuevo

He copiado de páginas web fragmentos de textos $\mathrm{y}$, sin citar, los he incorporado al trabajo que tenía escrito

He copiado fragmentos de fuentes impresas (libros, periódicos, artículos de revista, etc.) y , sin citarlos, los he incorporado al trabajo que tenía escrito

He entregado como propio algún trabajo completo descargado de Internet, sin modificarlo

He hecho íntegramente algún trabajo a partir de fragmentos copiados literalmente de páginas web

He hecho íntegramente algún trabajo a partir de fuentes impresas, sin poner los/as autores/as

He utilizado fragmentos de los apuntes del profesor para elaborar algún trabajo, sin citarlos

.085

.085

.146

.091

.119

.099

.067

.099

.302

.418

.505

.502

.229

.382

.392

.440

440

\section{CAUSAS QUE TE HAN MOTIVADO A REALIZAR LAS ACCIONES ANTERIORES}

Es un "atajo" aceptado por todos/as

Mis compañeros/as lo hacen

Lo considero un desafío al profesorado, a la autoridad

Es fácil y cómodo el acceso a material vía Internet

Me permite obtener mejores resultados académicos

.571
.440

.156

.549

Desconocía la existencia de normativa en mi universidad que lo penalice

Las sanciones no son graves

Desconocía que tenía que citar siempre

Falta de instrucciones precisas sobre cómo hacer el trabajo

Falta de motivación

Falta de tiempo

Sobrecarga de trabajo

Lo que está en Internet es de propiedad común

\section{EL PROFESORADO}

Me da instrucciones precisas para elaborar el trabajo

Me facilita las normas básicas para citar adecuadamente

Realiza un seguimiento de la evolución del trabajo teniendo en cuenta la citación de fuentes

Ofrece atención individualizada en el aula durante la elaboración del trabajo

Ofrece atención individualizada en las tutorías durante la elaboración del trabajo

Evalúa la correcta citación de las fuentes documentales incorporadas

Pide trabajos esencialmente teóricos

Pide trabajos esencialmente prácticos

Maneja herramientas de detección de fragmentos copiados en los trabajos

Adecúa la carga de trabajos al tiempo disponible para cada asignatura

Se coordina con los demás docentes para evitar saturar de trabajos al alumnado

$.538 \quad .873$

$.304 \quad .877$

$.284 \quad .877$

$.394 \quad .875$

$.519 \quad .873$

$.496 \quad .873$

$.383 \quad .876$

.112

.134

.125

.132

177

.147

.143

.127

.074

.139

.093
.880

.880

.879

.880

.879

.879

.880

.880

.877

.875

.874

.874

.878

.876

,876

.875

$.323 \quad .877$

$.470 \quad .874$

.873

.875

873

877

873

876

.879

.879

.879

.879

.879

.879

.879

.879

.880

.879

.879 


\begin{tabular}{|c|c|c|}
\hline & $\begin{array}{c}\text { Correlación total } \\
\text { de elementos } \\
\text { corregida }\end{array}$ & $\begin{array}{c}\alpha \text { de Cronbach } \\
\text { si se suprime el } \\
\text { item }\end{array}$ \\
\hline \multicolumn{3}{|c|}{ CREO QUE MIS COMPAÑEROS/AS } \\
\hline Han entregado un trabajo realizado por un/a compañero/a en cursos anteriores & .489 & 874 \\
\hline $\begin{array}{l}\text { Han copiado partes de los trabajos que han entregado en cursos anteriores para } \\
\text { uno nuevo }\end{array}$ & .556 & .873 \\
\hline $\begin{array}{l}\text { Han copiado de páginas web fragmentos de textos y, sin citar, los han } \\
\text { incorporado al trabajo que tenían escrito }\end{array}$ & .627 & .871 \\
\hline $\begin{array}{l}\text { Han copiado fragmentos de fuentes impresas (libros, periódicos, artículos de } \\
\text { revista, etc.) y, sin citarlos, los han incorporado al trabajo que tenían escrito }\end{array}$ & .601 & .872 \\
\hline $\begin{array}{l}\text { Han entregado un trabajo completo descargado de Internet, sin modificarlo, } \\
\text { como propio }\end{array}$ & .465 & .874 \\
\hline $\begin{array}{l}\text { Han hecho íntegramente un trabajo a partir de fragmentos copiados } \\
\text { literalmente de páginas web }\end{array}$ & .548 & .873 \\
\hline Han hecho íntegramente un trabajo a partir de fuentes impresas & .507 & 874 \\
\hline $\begin{array}{l}\text { Han utilizado fragmentos de los apuntes del profesor para elaborar un trabajo, } \\
\text { sin citarlos }\end{array}$ & .521 & .873 \\
\hline Copian más en trabajos de aula que en el TFG/TFM & .423 & .875 \\
\hline $\begin{array}{l}\text { Admiten como apropiado el método de “cortar” y “pegar” al presentar un } \\
\text { trabajo }\end{array}$ & .531 & .873 \\
\hline
\end{tabular}

De este modo, tras la eliminación de dichos ítems, el cuestionario queda reducido a un total de 30 frente a los 50 iniciales y a tres dimensiones A lo largo de la carrera... (8 ítems), Causas que te han motivado a realizar las acciones anteriores (12 ítems) y Creo que mis compañeros/as... (10 ítems). Esta reducción considerable en el número de ítems guarda coherencia con el marco teórico sobre el plagio puesto que las dimensiones e ítems que han sido eliminados, si bien tienen relación con el constructo a estudiar, no aluden específicamente al plagio, sino a otros elementos relacionados, tales como las acciones para no cometer plagio y la importancia de citar.

Con esta nueva configuración, se procedió al cálculo de la fiabilidad del instrumento. En este caso, el coeficiente Alfa de Cronbach es de .924, lo que supone un incremento en dos puntos respecto al análisis de fiabilidad previo. Al estudiar los índices de homogeneidad de todos los ítems (tabla 3), se comprueba que todos tienen puntuaciones superiores a .2, alcanzando la mayoría de las correlaciones valores próximos o superiores a .5 , lo que evidencia que el cuestionario, una vez realizadas las modificaciones previas, tiene una buena consistencia interna. 
Muñoz-Cantero, Jesús-Miguel; Rebollo-Quintela, Nuria; Mosteiro-García, Josefa, \& Ocampo-Gómez, Camilo-Isaac (2019). Validación del cuestionario de atribuciones para la detección de coincidencias en trabajos académicos. RELIEVE, 25(1), art. 4. doi: http://doi.org/10.7203/relieve.25.1.13599

Tabla 3 - Valores de los índices de homogeneidad de cada ítem y del índice de fiabilidad del cuestionario tras la eliminación de los ítems

\begin{tabular}{|c|c|c|}
\hline & $\begin{array}{l}\text { Correlación } \\
\text { total de } \\
\text { elementos } \\
\text { corregida }\end{array}$ & $\begin{array}{l}\text { ade } \\
\text { Cronbach si } \\
\text { el elemento se } \\
\text { ha suprimido }\end{array}$ \\
\hline \multicolumn{3}{|l|}{ A LO LARGO DE LA CARRERA } \\
\hline He entregado algún trabajo realizado por otros/as en cursos anteriores & .329 & .924 \\
\hline $\begin{array}{l}\text { He copiado partes de trabajos entregados en cursos anteriores para un } \\
\text { trabajo nuevo }\end{array}$ & .476 & .923 \\
\hline $\begin{array}{l}\text { He copiado de páginas web fragmentos de textos y, sin citar, los que } \\
\text { incorporado al trabajo que tenía escrito }\end{array}$ & .594 & .921 \\
\hline $\begin{array}{l}\text { He copiado fragmentos de fuentes impresas (libros, periódicos, artículos de } \\
\text { revista, etc.) y, sin citarlos, los he incorporado al trabajo que tenía escrito }\end{array}$ & .574 & .921 \\
\hline $\begin{array}{l}\text { He entregado como propio algún trabajo completo descargado de Internet, } \\
\text { sin modificarlo }\end{array}$ & .238 & .925 \\
\hline $\begin{array}{l}\text { He hecho íntegramente algún trabajo a partir de fragmentos copiados } \\
\text { literalmente de páginas web }\end{array}$ & .438 & .923 \\
\hline $\begin{array}{l}\text { He hecho íntegramente algún trabajo a partir de fuentes impresas, sin poner } \\
\text { los/as autores/as }\end{array}$ & .432 & .923 \\
\hline $\begin{array}{l}\text { He utilizado fragmentos de los apuntes del profesor para elaborar algún } \\
\text { trabajo, sin citarlos }\end{array}$ & .496 & .922 \\
\hline \multicolumn{3}{|c|}{ CAUSAS QUE TE HAN MOTIVADO A REALIZAR LAS ACCIONES ANTERIORES } \\
\hline Es un "atajo" aceptado por todos/as & .656 & .920 \\
\hline Mis compañeros/as lo hacen & .466 & .923 \\
\hline Es fácil y cómodo el acceso a material vía Internet & .604 & .921 \\
\hline Me permite obtener mejores resultados académicos & .609 & .921 \\
\hline Desconocía la existencia de normativa en mi universidad que lo penalice & .343 & .924 \\
\hline Las sanciones no son graves & .318 & .924 \\
\hline Desconocía que tenía que citar siempre & .390 & .924 \\
\hline Falta de instrucciones precisas sobre cómo hacer el trabajo & .523 & .922 \\
\hline Falta de motivación & .590 & .921 \\
\hline Falta de tiempo & .622 & .920 \\
\hline Sobrecarga de trabajo & .608 & .921 \\
\hline Lo que está en Internet es de propiedad común & .465 & .923 \\
\hline \multicolumn{3}{|l|}{ CREO QUE MIS COMPAÑEROS/AS } \\
\hline $\begin{array}{l}\text { Han entregado un trabajo realizado por un/a compañero/a en cursos } \\
\text { anteriores }\end{array}$ & .531 & .922 \\
\hline $\begin{array}{l}\text { Han copiado partes de los trabajos que han entregado en cursos anteriores } \\
\text { para uno nuevo }\end{array}$ & .598 & .921 \\
\hline $\begin{array}{l}\text { Han copiado de páginas web fragmentos de textos y, sin citar, los han } \\
\text { incorporado al trabajo que tenían escrito }\end{array}$ & .677 & .920 \\
\hline $\begin{array}{l}\text { Han copiado fragmentos de fuentes impresas (libros, periódicos, artículos de } \\
\text { revista, etc.) y , sin citarlos, los han incorporado al trabajo que tenían } \\
\text { escrito }\end{array}$ & .641 & .920 \\
\hline $\begin{array}{l}\text { Han entregado un trabajo completo descargado de Internet, sin modificarlo, } \\
\text { como propio }\end{array}$ & .481 & .922 \\
\hline $\begin{array}{l}\text { Han hecho íntegramente un trabajo a partir de fragmentos copiados } \\
\text { literalmente de páginas web }\end{array}$ & .583 & .921 \\
\hline Han hecho íntegramente un trabajo a partir de fuentes impresas & .527 & .922 \\
\hline $\begin{array}{l}\text { Han utilizado fragmentos de los apuntes del profesor para elaborar un } \\
\text { trabajo, sin citarlos }\end{array}$ & .549 & .922 \\
\hline Copian más en trabajos de aula que en el TFG/TFM & .451 & .923 \\
\hline $\begin{array}{l}\text { Admiten como apropiado el método de “cortar” y “pegar” al presentar un } \\
\text { trabajo }\end{array}$ & .574 & .921 \\
\hline
\end{tabular}




\section{Análisis factorial exploratorio}

Con el objeto de estimar la validez de constructo del cuestionario, se llevó a cabo un análisis factorial con extracción de componentes principales y posterior rotación Varimax.

Previamente a la realización del análisis factorial, se procedió a la aplicación de la prueba de Kayser-Meyer-Olsen (KMO) y de la Prueba de Esfericidad de Barlett, para comprobar la adecuación muestral de los indicadores del cuestionario (Muñoz Cantero, Casar Domínguez, \& Abalde Paz, 2007). El valor próximo a 1 de la prueba $\mathrm{KMO}=.916 \mathrm{y}$ el resultado estadísticamente significativo de la prueba de esfericidad de Bartlett: $\chi_{435}^{2}=$ $31680.841 \quad(\mathrm{p}<.001)$ confirman la pertinencia de realizar el análisis factorial, que se realizó posteriormente con el objeto de determinar la estructura dimensional del cuestionario y comprobar si coincide con la estructura postulada a nivel teórico (Bryman, 2016). Para la extracción de factores se ha hecho uso del método de extracción de componentes principales, método iterativo que como señalan De Winter \& Dodou (2012) se basa en la extracción sucesiva de factores que explican la mayor parte de la varianza común.

Con el fin de obtener una solución factorial lo más simple posible, se utilizó como método de rotación varimax, de carácter ortogonal que minimiza el número de variables que tienen un factor o componente de saturación sobre una variable acentuando así las que lo tienen más elevado.

Como se puede observar, en la tabla 4, en la que se recogen los porcentajes de la varianza total del modelo antes y después de la rotación, no se han producido cambios en la varianza total explicada para los cinco primeros componentes, siendo en ambos casos del 59.164\%, con valores superiores a 1. No obstante, sí se producen modificaciones en la parte explicada de manera individual por cada componente o factor, ya que, a raíz de la rotación el 31.919\% correspondiente al primer factor sin rotar, pasa a ser del $19.667 \%$, el $11.965 \%$ del segundo factor al $15.761 \%$, el $6.391 \%$ frente al $8.934 \%$ del tercer factor y el $4.732 \%$ y $4.157 \%$ del cuarto y quinto factor pasa al $8.722 \%$ y $6.080 \%$ de la matriz rotada respectivamente.

Tabla 4 - Varianza total explicada. Método de extracción: Análisis de Componentes principales

\begin{tabular}{crrrrrrrrr}
\hline \multirow{2}{*}{ Comp. } & \multicolumn{4}{c}{ Autovalores iniciales } & \multicolumn{2}{c}{$\begin{array}{c}\text { Sumas de extracción de } \\
\text { cargas al cuadrado }\end{array}$} & \multicolumn{2}{c}{$\begin{array}{c}\text { Sumas de rotación de cargas } \\
\text { al cuadrado }\end{array}$} \\
\cline { 2 - 11 } & Total & $\begin{array}{c}\text { Porcentaje } \\
\text { varianza }\end{array}$ & $\begin{array}{c}\text { Porcentaje } \\
\text { acumulado }\end{array}$ & Total & $\begin{array}{c}\text { Porcentaje } \\
\text { varianza }\end{array}$ & $\begin{array}{c}\text { Porcentaje } \\
\text { acumulado }\end{array}$ & Total & $\begin{array}{c}\text { Porcentaje } \\
\text { varianza }\end{array}$ & $\begin{array}{c}\text { Porcentaje } \\
\text { acumulado }\end{array}$ \\
\hline 1 & 9.576 & 31.919 & 31.919 & 9.576 & 31.919 & 31.919 & 5.900 & 19.667 & 19.667 \\
2 & 3.590 & 11.965 & 43.884 & 3.590 & 11.965 & 43.884 & 4.728 & 15.761 & 35.428 \\
3 & 1.917 & 6.391 & 50.275 & 1.917 & 6.391 & 50.275 & 2.680 & 8.934 & 44.362 \\
4 & 1.420 & 4.732 & 55.007 & 1.420 & 4.732 & 55.007 & 2.617 & 8.722 & 53.084 \\
5 & 1.247 & 4.157 & 59.164 & 1.247 & 4.157 & 59.164 & 1.824 & 6.080 & 59.164 \\
\hline
\end{tabular}

El análisis de las comunalidades, muestra que la mayoría de los ítems están bien explicados por la estructura factorial, a excepción de $\mathrm{He}$ entregado algún trabajo realizado por otros/as en cursos anteriores (comunalidad $=.462)$, Las sanciones no son graves (comunalidad=.297), Lo que está en Internet es de propiedad común (comunalidad=.430), Copian más en trabajos de aula que en el TFG/TFM (comunalidad=.422) que tienen valores inferiores a .50. Estos cinco ítems son eliminados pues carecen de una explicación suficiente y no deberían ser considerados en la interpretación final del análisis (Zamora, Monroy, \& Chávez, 2009), quedando el cuestionario configurado por un total de 26 ítems, se llevó a cabo un segundo análisis factorial para confirmar su comportamiento en las dimensiones establecidas. 
Tabla 5 - Método de extracción: Análisis de Componentes principales. Ítems con comunalidades inferiores a .50

Inicial Extracción

\begin{tabular}{lcc}
\hline He entregado algún trabajo realizado por otros/as en cursos anteriores & 1.000 & .462 \\
\hline Las sanciones son graves & 1.000 & .297 \\
\hline Lo que está en Internet es de propiedad común & 1.000 & .430 \\
\hline Copian más en trabajos de aula que en el TFG/TFM & 1.000 & .422 \\
\hline
\end{tabular}

Al igual que en el primer análisis factorial se aprecian valores suficientemente solventes tanto en la prueba de adecuación muestral $\mathrm{KMO}=.907$, algo inferior al análisis anterior, que mostraba un valor de .916, como en la prueba de esfericidad de Bartlett: $\chi_{325}^{2}=29590.124(\mathrm{p}<.001)$.

El análisis factorial exploratorio supuso el mantenimiento del número de factores, cinco, con valores propios superiores a 1 , que son capaces de explicar un $63.351 \%$ de la variabilidad total lo que puede considerarse como un valor aceptable y superior al primer análisis factorial. Se puede observar que, al igual que en el primer análisis factorial y como consecuencia de la rotación, el porcentaje de la varianza total explicada se mantiene y solo se producen cambios en la parte explicada de manera individual por cada componente o factor, es decir, disminuye el peso del primer y segundo factor y aumenta el de los tres últimos (tabla 6). Tras estudiar nuevamente el valor de la comunalidad de los ítems, ahora todas ellas son superiores a $.50 \mathrm{y}$ por tanto ningún factor ve afectada su conveniencia o idoneidad para su presentación definitiva.

Tabla 6 - Varianza total explicada. Método de extracción: Análisis de Componentes principales

\begin{tabular}{|c|c|c|c|c|c|c|c|c|c|}
\hline \multirow[t]{2}{*}{ Comp. } & \multicolumn{3}{|c|}{ Autovalores iniciales } & \multicolumn{6}{|c|}{$\begin{array}{c}\text { Sumas de extracción de cargas Sumas de rotación de cargas al } \\
\text { al cuadrado }\end{array}$} \\
\hline & Total & $\begin{array}{l}\text { Porcentaje } \\
\text { varianza }\end{array}$ & $\begin{array}{l}\text { Porcentaje } \\
\text { acumulado }\end{array}$ & Total & $\begin{array}{c}\text { Porcentaje } \\
\text { varianza }\end{array}$ & $\begin{array}{l}\text { Porcentaje } \\
\text { acumulado }\end{array}$ & Total & $\begin{array}{l}\text { Porcentaje } \\
\text { varianza }\end{array}$ & $\begin{array}{l}\text { Porcentaje } \\
\text { acumulado }\end{array}$ \\
\hline 1 & 8.81 & 33.897 & 33.897 & 8.81 & 33.897 & 33.897 & 5.454 & 20.976 & 20.976 \\
\hline 2 & 01 & 12.697 & 46.594 & 3.301 & 12.697 & 46.594 & 4.230 & 16.270 & 37.246 \\
\hline 3 & 1.815 & 6.979 & 53.573 & 15 & 6.979 & 53.573 & 2.673 & 10.281 & 47.527 \\
\hline 4 & 1.357 & 5.220 & 58.793 & 1.357 & 5.220 & 58.793 & 2.270 & 8.731 & 56.258 \\
\hline 5 & 1.185 & 4.559 & 63.351 & 1.185 & 4.559 & 63.351 & 1.844 & 7.094 & 63.351 \\
\hline
\end{tabular}

La representación gráfica de la estructura factorial en cinco factores y los valores propios (figura 1), muestra que el cambio de pendiente de la curva se produce a partir del quinto factor, acumulando el $63.351 \%$ de la varianza total. 


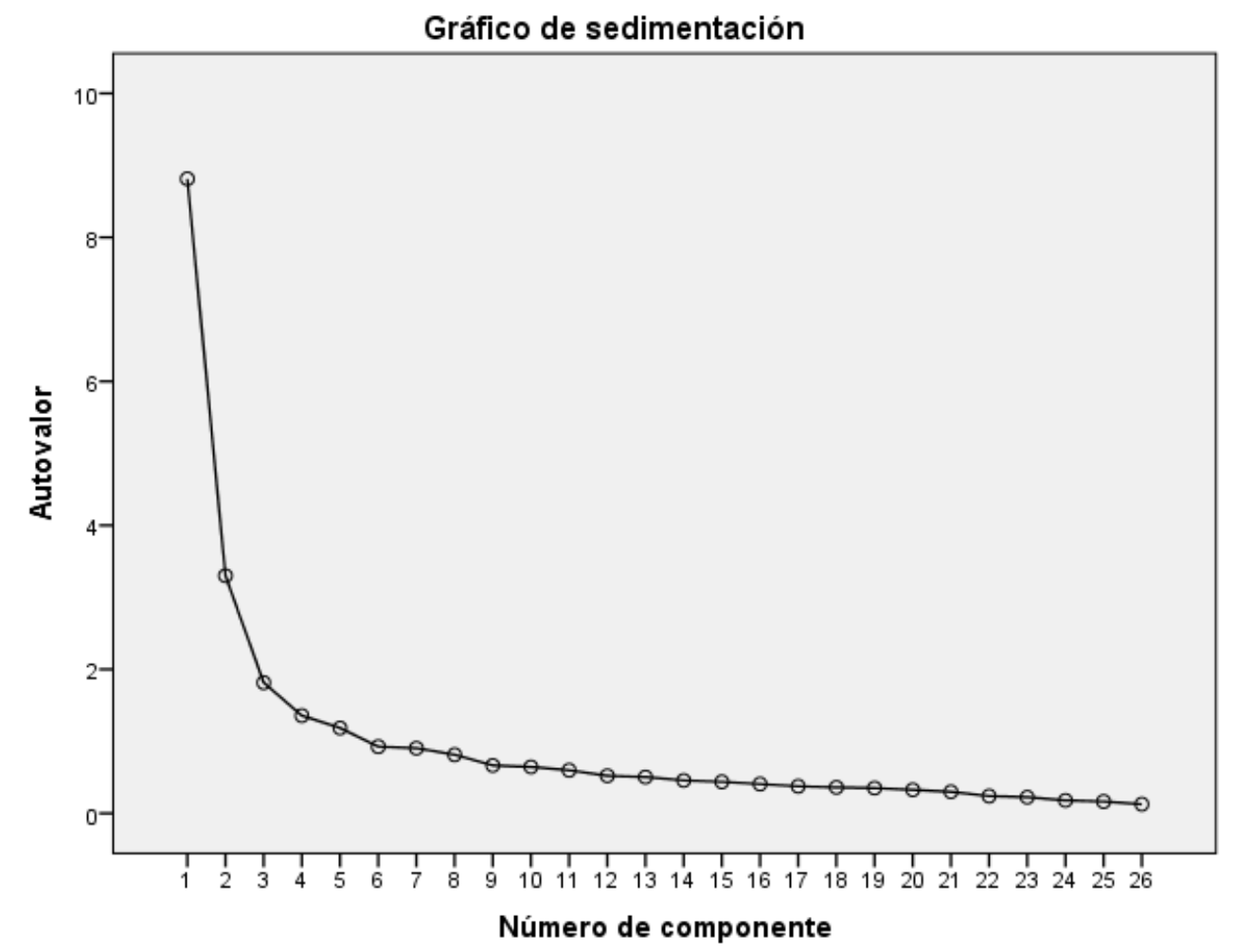

Figura 1. Representación gráfica de la sedimentación de los autovalores en función de cada uno de los componentes expuestos correspondiente al análisis factorial inicial

El modelo factorial está formado por buenos indicadores del constructo a estudiar, ya que como podemos comprobar no se aprecia ningún ítem con cargas factoriales en valor absoluto de .40 (tabla 7).

El primer factor explica el $20.976 \%$ e incluye un total de nueve ítems y en él se agrupan todos los que estaban contemplados en el cuestionario inicial bajo la denominación Creo que mis compañeros/as, a excepción del ítem Copian más en trabajos de aula que en el TFG/TFM eliminado en el análisis previo, relacionados con la opinión del estudiantado acerca de la realización de prácticas deshonestas por parte de sus compañeros y compañeras, es decir, las actitudes del grupo de iguales hacia el plagio.

El segundo explica un $16.270 \%$ de la varianza y comprende siete ítems: Es un "atajo" aceptado por todos/as; Mis compañeros/as lo hacen; Es fácil y cómodo el acceso a material vía Internet; Me permite obtener mejores resultados académicos; Falta de motivación; Falta de tiempo y Sobrecarga de trabajo, correspondientes a la dimensión causas que te han motivado a realizar las acciones anteriores, que atendiendo a su contenido se podrían agrupar bajo la dimensión causas internas del plagio.

El tercer factor agrupa cuatro ítems que aluden al plagio parcial y explica el $10.281 \%$ de la varianza, se corresponden con el bloque del cuestionario inicial A lo largo de la carrera... He copiado partes de trabajos entregados en cursos anteriores para un trabajo nuevo; He copiado de páginas web fragmentos de textos $y$, sin citar, los he incorporado al trabajo que tenía escrito; He copiado fragmentos de fuentes impresas (libros, periódicos, artículos de revista etc.) y, sin citarlos, los he incorporado al trabajo que tenía escrito y He utilizado fragmentos de los apuntes del profesor para elaborar algún trabajo sin citarlos.

El cuarto que explica el $8.731 \%$ de la varianza está compuesto por tres ítems respecto al plagio total, bloque A lo largo de la carrera... He entregado como propio algún trabajo completo descargado de Internet, sin modificarlo; He hecho íntegramente algún trabajo a partir de fragmentos copiados literalmente de páginas 
web y He hecho íntegramente algún trabajo a partir de fuentes impresas, sin poner los/as autores/as.

El quinto factor, que explica el $7.094 \%$ de la varianza, agrupa algunos ítems del bloque Causas que te han motivado a realizar las acciones anteriores: Desconocía la existencia de normativa en mi universidad que lo penalice; Desconocía que tenía que citar siempre y Falta de instrucciones precisas sobre cómo hacer el trabajo. Estos ítems se pueden englobar bajo la categoría causas externas del plagio.

Tabla 7 - Método de extracción: Análisis de componentes principales a 5 componentes extraídos

\begin{tabular}{|c|c|c|c|c|c|}
\hline & \multicolumn{5}{|c|}{ COMPONENTE } \\
\hline & 1 & 2 & 3 & 4 & 5 \\
\hline $\begin{array}{l}\text { He copiado partes de trabajos entregados en cursos anteriores para } \\
\text { un trabajo nuevo }\end{array}$ & & & .353 & & \\
\hline $\begin{array}{l}\text { He copiado de páginas web fragmentos de textos y, sin citar, los } \\
\text { que incorporado al trabajo que tenía escrito }\end{array}$ & & & .718 & & \\
\hline $\begin{array}{l}\text { He copiado fragmentos de fuentes impresas (libros, periódicos, } \\
\text { artículos de revista, etc.) y, sin citarlos, los he incorporado al } \\
\text { trabajo que tenía escrito }\end{array}$ & & & .715 & & \\
\hline $\begin{array}{l}\text { He entregado como propio algún trabajo completo descargado de } \\
\text { Internet, sin modificarlo }\end{array}$ & & & & .726 & \\
\hline $\begin{array}{l}\text { He hecho íntegramente algún trabajo a partir de fragmentos } \\
\text { copiados literalmente de páginas web }\end{array}$ & & & & .724 & \\
\hline $\begin{array}{l}\text { He hecho íntegramente algún trabajo a partir de fuentes impresas, } \\
\text { sin poner los/as autores/as }\end{array}$ & & & & .648 & \\
\hline $\begin{array}{l}\text { He utilizado fragmentos de los apuntes del profesor para elaborar } \\
\text { algún trabajo, sin citarlos }\end{array}$ & & & 666 & & \\
\hline Es un "atajo" aceptado por todos/as & & .608 & & & \\
\hline Mis compañeros/as lo hacen & & .495 & & & \\
\hline Es fácil y cómodo el acceso a material vía Internet & & .606 & & & \\
\hline Me permite obtener mejores resultados académicos & & .573 & & & \\
\hline $\begin{array}{l}\text { Desconocía la existencia de normativa en mi universidad que lo } \\
\text { penalice }\end{array}$ & & & & & .707 \\
\hline Desconocía que tenía que citar siempre & & & & & .805 \\
\hline Falta de instrucciones precisas sobre cómo hacer el trabajo & & & & & .579 \\
\hline Falta de motivación & & .797 & & & \\
\hline Falta de tiempo & & .845 & & & \\
\hline Sobrecarga de trabajo & & .842 & & & \\
\hline $\begin{array}{l}\text { Han entregado un trabajo realizado por un/a compañero/a en } \\
\text { cursos anteriores }\end{array}$ & .768 & & & & \\
\hline $\begin{array}{l}\text { Han copiado partes de los trabajos que han entregado en cursos } \\
\text { anteriores para uno nuevo }\end{array}$ & .785 & & & & \\
\hline $\begin{array}{l}\text { Han copiado de páginas web fragmentos de textos y, sin citar, los } \\
\text { han incorporado al trabajo que tenían escrito }\end{array}$ & .796 & & & & \\
\hline $\begin{array}{l}\text { Han copiado fragmentos de fuentes impresas (libros, periódicos, } \\
\text { artículos de revista, etc.) y, sin citarlos, los han incorporado al } \\
\text { trabajo que tenían escrito }\end{array}$ & .788 & & & & \\
\hline $\begin{array}{l}\text { Han entregado un trabajo completo descargado de Internet, sin } \\
\text { modificarlo, como propio }\end{array}$ & .742 & & & & \\
\hline $\begin{array}{l}\text { Han hecho íntegramente un trabajo a partir de fragmentos } \\
\text { copiados literalmente de páginas web }\end{array}$ & .821 & & & & \\
\hline Han hecho íntegramente un trabajo a partir de fuentes impresas & .753 & & & & \\
\hline $\begin{array}{l}\text { Han utilizado fragmentos de los apuntes del profesor para } \\
\text { elaborar un trabajo, sin citarlos }\end{array}$ & .629 & & & & \\
\hline $\begin{array}{l}\text { Admiten como apropiado el método de “cortar” y “pegar” al } \\
\text { presentar un trabajo }\end{array}$ & .704 & & & & \\
\hline
\end{tabular}




\section{Discusión}

Las prácticas deshonestas, como fenómeno de magnitud internacional, han sido tema de numerosas investigaciones en las últimas décadas, centrando sus esfuerzos en el estudio del plagio como actividad fraudulenta de mayor prevalencia. Como hemos podido observar, la notable repercusión manifiesta, viene dada en gran medida por sus implicaciones legales, éticas y educativas.

En el campo objeto de estudio, la revisión de la literatura muestra la existencia de diferentes instrumentos centrados en el plagio, que pretenden delimitar los diversos aspectos de un fenómeno multidimensional, si bien, no existe unanimidad en los factores clave sobre los que se asienta la medición del constructo. Sin embargo, los estudios publicados coinciden en las importantes implicaciones del plagio en la formación del alumnado universitario. De hecho, es creciente la reivindicación a voces de la necesidad de una formación ética como aspecto inherente a los planes de estudio y a los contextos de aprendizaje universitarios. Martínez \& Esteban (2005), señalan que "el aprendizaje de competencias éticas es especialmente relevante en el aprendizaje de la ciudadanía y, en especial, en el aprendizaje de la ciudadanía para la inclusión social” (p. 75).

Partiendo de estas consideraciones, surge la necesidad de diseñar instrumentos capaces de analizar con una perspectiva holística este fenómeno. Para ello, es determinante poner el punto de vista en el alumnado, ya que la evaluación de su percepción es "el primer paso para entender la situación y planificar programas educativos para reducir y prevenir la comisión de plagio" (Poorolajal et al., 2012, p.57).

En este artículo, se ha realizado la construcción del Cuestionario de atribuciones para la detección de coincidencias en trabajos académicos (CUDECO), basado en el análisis exhaustivo de diferentes instrumentos validados (Cebrián-Robles et al., 2018; Comas-Forgas \& Sureda-Negre, 2010; Ehrich et al., 2016; Mavrinac et al., 2010). Como resultado de dicho proceder se elaboró el cuestionario inicial, que tras ser sometido a juicio de expertos, quedó configurado definitivamente por un total 59 preguntas, 9 referidas a las variables de identificación del sujeto y 39 ítems de escala tipo Likert agrupados en cinco dimensiones ¿Qué utilidad tiene para ti citar?, A lo largo de la carrera..., Causas que te han motivado a realizar las acciones anteriores, El profesorado... y Creo que mis compañeros/as...

Atendiendo a los objetivos propuestos se procedió al estudio de la fiabilidad y validez del instrumento a fin de determinar su idoneidad. El resultado del análisis de consistencia interna y de los estadísticos de fiabilidad para los elementos que lo componen ha llevado a la modificación del instrumento original que quedó configurado por un total de 28 ítems, presentando tras este reajuste una elevada fiabilidad.

Por su parte, la estructura mediante el análisis factorial exploratorio muestra un modelo de cinco factores que hacen referencia al concepto de plagio y sus tipos (parcial y total), las causas que motivan la comisión del mismo tanto internas (propias del sujeto) como externas (ajenas al sujeto) y las actitudes del grupo de iguales hacia el plagio, hallazgos en la línea de investigaciones previas (Bennett, 2005; Cebrián-Robles et al. 2018; Comas-Forgas \& Sureda-Negre, 2010; Mavrinac et al., 2010). Así mismo, los factores resultantes han sido abordados en trabajos de corte cualitativa como Ashworth, Bannister, \& Thorne (1997), a través de entrevistas; Ochoa \& Cueva (2016) en cuestionarios abiertos y Devlin \& Gray (2007) y Gullifer \& Tyson (2010) mediante grupos de discusión. Sin embargo, este cuestionario ofrece una visión más integradora que los instrumentos previos en cuanto a las dimensiones estudiadas, analizando no solamente los factores internos y externos que motivan la comisión de plagio, sino que nos permite conocer cuál es la percepción respecto al papel que el profesorado juega en el proceso, habida 
cuenta que, desde el cambio de paradigma como consecuencia del desarrollo del Espacio Europeo de Educación Superior, no se ha analizado esta variable. Por otro lado, la visión aportada desde este cuestionario es en cierta medida innovadora en este tipo de estudios, al verificar que la percepción de los sujetos respecto a sus pares es una dimensión válida y fiable para poder explicar el problema.

Los resultados obtenidos confirman la validez de un instrumento, útil y fiable para la detección de coincidencias en trabajos en el contexto universitario. Por otro lado, se considera una herramienta valiosa para llevar a cabo un diagnóstico de las conductas de plagio por parte del alumnado, así como para determinar las causas subyacentes que motivan a cometerlo. Todo ello, posibilitará la adopción de medidas de carácter preventivo dirigidas a que estas conductas deshonestas dejen de ser una práctica habitual.

Por último, sería de interés en futuras investigaciones, ampliar el campo de actuación, y abarcar las etapas de Educación Secundaria y Bachillerato, a fin de determinar la génesis del plagio desde sus primeras manifestaciones. Así mismo, sería conveniente adaptar y validar el instrumento para esta población lo que permitirá comprobar en qué medida los ítems se ajustan a la dimensionalidad propuesta para estudiar el constructo de referencia en otras realidades diferentes (Bryman, 2016).

\section{Referencias}

Akbulut, Y., Sendag, S., Birinci, G., Kiliçer, K., Sahin, M., \& Odabasi, H.F. (2008). Exploring the types and reasons of Interettriggered academic dishonesty among Turkish undergraduate students: Development of Internet-Triggered Academic Dishonesty Scale (ITADS). Computers \& Education 51, 463-473. doi: https://doi.org/10.1016/j.compedu.2007.06.0 $\underline{03}$

Amiri, F., \& Razmjoo, S. A. (2016). On Iranian EFL Undergraduate Students' Perceptions of Plagiarism. J Acad. Ethics,
14 ,

115-131.

doi: https://doi.org/10.1007/s10805-015-9245-3

Ashworth, P., Bannister, P., \& Thorne, P. (1997). Guilty in whose eyes? University students' perceptions of cheating and plagiarism in academic work and assessment. Studies in Higher Education, 22 (2), 187-203. doi: https://doi.org/10.1080/03075079712331381 $\underline{034}$

Bennett, R. (2005). Factors associated with student plagiarism in a post-1992 university. Assessment \& Evaluation in Higher Education, 30 (2), 137-162. doi: https://doi.org/10.1080/02602930420002642 $\underline{44}$

Bilic-Zulle L., Frkovic V., Turk T., Azman J., \& Petrovecki M. (2005). Prevalence of plagiarism among medical students. Croat Med J, 46 (1), 126-131. Recuperado de http://neuron.mefst.hr/docs/CMJ/issues/2005 /46/1/15726686.pdf

Bretag, T., \& Mahmud, S. (2009). Selfplagiarism or appropriate textual re-use? Journal of Academic Ethics, 7, 193-205. doi: https://doi.org/10.1007/s10805-0099092-1

Bryman. A. (2016). Social research methods $\left(6^{a}\right.$ ed.). Nueva York: Oxford University Press. doi: https://doi.org/10.17159/22214070/2016/v5i2a1

Cebrián-Robles, V., Raposo-Rivas, M., Cebrián-de-la-Serna, M., \& SarmientoCampos, J.A. (2018). Percepción sobre el plagio académico de estudiantes universitarios españoles. Educación $X X 1,21(2), \quad 105-129 . \quad$ doi: https://doi.org/10.5944/educxx1.20062

Cheung, K.Y.F., Stupple, E.J.N., \& Elander, J. (2017). Development and validation of Student Attitudes and Beliefs about Authorship Scale: a psychometrically robust measure of authorial identity. Studies in Higher Education, 42(1), 97-114. doi: https://doi.org/10.1080/03075079.2015.1034 $\underline{673}$ 
Comas-Forgas, R., \& Sureda-Negre,J. (2010). Academic plagiarism: explanatory factors from students' perspective. Journal Academic Ethics, 8, 217-232. doi: https://doi.org/10.1007/s10805-010-9121-0

Cronbach, L. J. (1951). Coefficient alpha and the internal structure of tests. Psychometrika, 16, 297-334. doi: https://doi.org/10.1007/BF02310555

De Winter. J., \& Dodou. D. (2012). Factor recovery by principal axis factoring and maximum likelihood factor analysis as a function of factor pattern and sample size. Journal of Applied Statistics,39(4), 695-710. doi:

http://doi.org/10.1080/02664763.2011.6104 $\underline{4}$

Devlin, M., \& Gray, K. (2007). In their own words: a qualitative study of the reasons Australian university students plagiarize. High Education Research \& Development, 26(2), 181-198. doi: https://doi.org/10.1080/07294360701310805

Ehrich, J., Howard, S. J., Mu, C., \& Bokosmaty, S. (2016). A comparison of Chinese and Australian university students' attitudes towards plagiarism. Studies in Higher Education, 41 (2), 231-246. doi: https://doi.org/10.1080/03075079.2014.9278 $\underline{50}$

Ferreira,M.M., \& Persike, A. (2018). As concepçoes brasilera e anglófona de plágio: um estudo preliminar. Signótica, Goiânia, $30 \quad$ (2), 149-181. doi: https://doi.org/10.5216/sig.v30i2.46558

Finn, K,V., \& Frone, M.R. (2004). Academic performance and cheating: moderating role of school identification and self-efficacy. The journal of educational research, 97 (3), 115-121. doi: https://doi.org/10.3200/JOER.97.3.115-121

Gómez- Espinosa, M., Francisco, V., \& Moreno-Ger, P. (2016). El impacto del diseño de actividades en el plagio de Internet en educación superior. Comunicar, 48, 39-48. doi: https://doi.org/10.3916/C482016-04
Guerrero Sánchez, P., Mercado Yebra, J., \& Ibarra, L.M. (2017). La deshonestidad, elemento que altera la integridad en las prácticas académicas en las Instituciones de Educación Superior. Estudios de caso comparados. Investigación y formación pedagógica Revista del CIEGC, 3(5), 6-25. Recuperado de http://revistas.upel.edu.ve/index.php/revinvf ormpedag/article/view/5183/2683

Gullifer, J., \& Tyson, G. A. (2010). Exploring university students' perceptions of plagiarism: a focus group study. Studies in Higher Education, 35(4), 463-481. doi: https://doi.org/10.1080/03075070903096508

Harris, R. A. (2001). The Plagiarism Handbook: Strategies for Preventing, Detecting, and Dealing with Plagiarism. Los Angeles, CA: Pyrczak.

Hawley, C.S. (1984). The thives of academe: plagiarism in the university system. Improving college and university teaching, $32 \quad$ (1), 35-39. doi: https://doi.org/10.1080/00193089.1984.1053 $\underline{3838}$

Howard, S. J., Ehrich, J. F., \& Walton, R. (2014). Measuring students' perceptions of plagiarism: Modification and Rasch validation of a plagiarism attitude scale. Journal of Applied Measurement, 15(4), 372-393. Recuperado de http://ro.uow.edu.au/sspapers/1461

Hu, G., \& Sun, X. (2016). Conocimientos y actitudes ante el plagio del profesorado de lengua inglesa en universidades chinas. Comunicar,48, 29-37. doi: https://doi.org/10.3916/C48-2016-03

Jereb E., Perc M., Lämmlein B., Jerebic J., Urh M., Podbregar I., \& Sprajc, P. (2018) Factors influencing plagiarism in higher education: A comparison of German and Slovene students. PLOS ONE, 13(8). doi: https://doi.org/10.1371/journal.pone.020225 $\underline{2}$

Martin, D.E., Rao, A., \& Sloan, L.R. (2011). Ethnicity, acculturation, and plagiarism: a criterion study of unethical academic 
conduct. Human organization, 70 (1). doi: https://doi.org/10.17730/humo.70.1.nl775v2 $\underline{\mathrm{u} 633678 \mathrm{k} 6}$

Martínez, M., \& Esteban, F. (2005). Una propuesta de formación ciudadana para el EEES. Revista española de pedagogía, 230, 63-84. Recuperado de https://revistadepedagogia.org/wpcontent/uploads/2007/06/230-04.pdf

Mavrinac, M., Brumini, G., Bilić-Zulle, L., \& Petrovečki, M. (2010). Construction and validation of attitudes toward plagiarism questionnaire. Croatian Medical Journal, 51 (3), 195-201. doi https://doi.org/10.3325/cmj.2010.51.195

Muñoz Cantero, J.M., Casar Domínguez, L.S., \& Abalde Paz, E. (2007). El “contexto" y las “metas y objetivos" como elementos clave en la calidad de la atención a la diversidad en centros no universitarios. RELIEVE, 13(2), 235-261. doi: https://doi.org/10.7203/relieve.13.2.4209

Ochoa S. L., \& Cueva L. A. (2016). Percepciones de estudiantes acerca del plagio: datos cualitativos. Revista Encuentros, 14(02), 25-41. doi: https://doi.org/10.15665/re.v14i2.822

Park, C. (2003). In Other (People's) Words: Plagiarism by University Students Literature and Lessons. Assessment \& Evaluation in Higher Education, 28(5), 471-88. doi: http://doi.org/10.1080/02602930320001203 $\underline{52}$

Pittam, G., Elander, J., Lusher, J. Fox, P., \& Payne, N. (2009). Student beliefs and attitudes about authorial identity in academic writing. Studies in Higher Education 34(2), 153-170. doi: https://doi.org/10.1080/03075070802528270
Poorolajal, J., Cheraghi,P., Irani,A.D., Cheragui, Z., \& Mirfakhraei,M. (2012). Construction of Knowledge, Attitude and Practice Questionnaire for Assessing Plagiarism. Iranian J Publ Health, 41(11), 54-58. Recuperado de http://ijph.tums.ac.ir

Popoveniuc, B. (2018). Plagiarism and the crisis of Higher Education. Revista Romaneasca pentru Educatie Multidimensionala, 10(3), 1-5. doi: https://doi.org/10.18662/rrem/57

Rebollo-Quintela, N., Espiñeira-Bellón, E.M., \& Muñoz-Cantero, J.M. (2017). Atribuciones causales en el plagio académico por parte de los estudiantes universitarios. Revista de Estudios $e$ Investigación en Psicología y Educación, Extr.(6), 192-196. doi: https://doi.org/10.17979/reipe.2017.0.06.245 $\underline{3}$

Sureda, J., Comas, R., \& Morey, M. (2009). Las causas del plagio académico entre el alumnado universitario según el profesorado. Revista Iberoamericana de Educación, 50, 197-220. Recuperado de http://redalyc.uaemex.mx/src/inicio/ArtPdfR ed.jsp?iCve $=80011741011$

Sureda-Negre, J., Comas- Forgas, R., \& Oliver-Trobat,M.F. (2015). Plagio académico entre alumnado de secundaria y bachillerato: Diferencias en cuanto al género y la procrastinación Comunicar, 44, 103111. doi: https://doi.org/10.3916/C44-2015$\underline{11}$

Zamora. S., Monroy. L., \& Chávez. C. (2009). Análisis Factorial: una técnica para valorar la dimensionalidad de la pruebas. Cuaderno Técnico $n^{\circ}$ 6. México: Centro Nacional de Evaluación para la Educación Superior. 


\section{Authors / Autores}

To know more

/ Saber más

Muñoz-Cantero, Jesús-Miguel (jesus.miguel.munoz@udc.es).

Profesor titular de universidad. Coordinador del Grupo de Investigación GIACE (Grupo de Investigación en Evaluación y Calidad Educativa). Su dirección postal es: Departamento de Didácticas específicas y Métodos de Investigación y Diagnóstico en Educación. Facultad de Ciencias de la Educación, Campus de Elviña, s/n 15071. Universidad de A Coruña (España).

Rebollo-Quintela, Nuria (nuria.rebollo@udc.es).

Profesora del Departamento de Didácticas Específicas y Métodos de Investigación y Diagnóstico en Educación en la Universidade da Coruña. Grupo de investigación GIACE (Grupo de Investigación en Evaluación y Calidad Educativa). Doctora internacional por la Universidade da Coruña. Es la autora de contacto para este artículo. Su dirección postal es: Dpto. Didácticas Específicas y Métodos de Investigación y Diagnóstico en Educación. Facultad de Ciencias de la Educación, Campus de Elviña, s/n 15071. Universidad de A Coruña (España).

\section{Mosteiro-García, Josefa (pepa.mosteiro@usc.es).}

Profesora contratada doctora del Área MIDE de la Universidad de Santiago de Compostela. Miembro del Grupo de investigación IDEA (Investigación, Diagnóstico Educativo y Evaluación). Dirección Postal: Departamento de Pedagogía y Didáctica. Facultad de Ciencias de la Educación. C/ Prof. Vicente Fráiz Andón, s/n, Campus Vida, 15782. Universidad de Santiago de Compostela (España).

\section{Ocampo-Gómez, Camilo-Isaac (cocampo@uvigo.es).}

Profesor Titular de Universidad. Miembro del grupo de Investigación Educativa GIE de la Universidad de Vigo. Dirección postal: Facultad de CC de la Educación. Máster de formación de profesorado de educación secundaria. Campus de Ourense de la Universidad de Vigo. 32004, Ourense (España).

\section{RELIEVE}

Revista ELectrónica de Investigación y EValuación Educativa E-Journal of Educational Research, Assessment and Evaluation

[ISSN: 1134-4032]

(C) Copyright, RELIEVE. Reproduction and distribution of this articles it is authorized if the content is no modified and their origin is indicated (RELIEVE Journal, volume, number and electronic address of the document).

(C) Copyright, RELIEVE. Se autoriza la reproducción y distribución de este artículo siempre que no se modifique el contenido y se indique su origen (RELIEVE, volumen, número y dirección electrónica del documento). 